\title{
非负特征图的线性染色
}

\section{王维凡*, 李超}

浙江师范大学数学系, 金华 321004

*E-mail: wwf@zjnu.cn

收稿日期: 2008-01-28; 接受日期: 2008-07-03

国家自然科学基金 (批准号: 10771197) 和浙江省自然科学基金 (批准号: Y607467) 资助项目

摘要 如果图 $G$ 的一个正常染色满足染任意两种颜色的顶点集合导出的子图是一些点 不交的路的并, 则称这个正常染色为图 $G$ 的线性染色. 图 $G$ 的线性色数用 $\operatorname{lc}(G)$ 表示, 是 指 $G$ 的所有线性染色中所用的最少颜色的个数.

证明了: 对于每一个最大度为 $\Delta(G)$ 围长为 $g(G)$ 的非负特征图 $G$, 若存在一个有序 对 $(\Delta, g) \in\{(13,7),(9,8),(7,9),(5,10),(3,13)\}$, 使得 $G$ 满足 $\Delta(G) \geqslant \Delta$ 且 $g(G) \geqslant g$, 则 $\operatorname{lc}(G)=\left\lceil\frac{\Delta(G)}{2}\right\rceil+1$.

关键词线性染色 非负特征图 围长 最大度 $\operatorname{MSC}(2000)$ 主题分类 $05 \mathrm{C} 15$

\section{1 引言}

本文所考虑的图都是简单图. 对于一个简单图 $G$, 我们用 $V(G), E(G),|G|, \Delta(G)$ 和 $\delta(G)$ 分别表示它的顶点集合、边集合、阶数、最大度和最小度. $G$ 的围长 $g(G)$ 是指 $G$ 中最短圈 的长度.

在文献 [1] 中, 图 $G$ 的一个正常染色是从顶点集合 $V(G)$ 到颜色集合 $\{1,2, \ldots, k\}$ 的一 个映射, 使得任意两个相邻的顶点染不同的颜色. 图 $G$ 的一个线性 $k$-染色是一个正常染色, 使得染任意两种颜色的顶点集合导出的子图是一些点不交的路的并. 图 $G$ 的线性色数 $\operatorname{lc}(G)$ 定义为 $G$ 的所有线性 $k$-染色中最小的 $k$ 值.

Yuster $^{[1]}$ 首先研究了图的线性染色, 证明了任意图 $G$ 的线性色数满足 $\operatorname{lc}(G)=O\left(\Delta^{\frac{3}{2}}\right)$, 且构造出了一类图, 使得 $\operatorname{lc}(G)=\Omega\left(\Delta^{\frac{3}{2}}\right)$. 事实上, 这个概念是图的无圈染色的一种特殊情 形. 图 $G$ 的一个无圈染色是 $G$ 的一个正常染色, 使得染任意两种颜色的顶点集合导出的子 图是一个森林. 无圈染色的概念是由 Grünbaum ${ }^{[2]}$ 提出的.

最近, Esperet 等 ${ }^{[3]}$ 把线性染色的概念推广到线性选择性上来. 他们研究了树、格子图、 完全二部图、平面图、外平面图、最大度为 3 或 4 的图以及有较小最大平均度的图的线性选 择数. 下面一些结果蕴含在他们的文章里:

定理 1 设 $G$ 是一个平面图. 
(1) 如果 $g(G) \geqslant 16$ 且 $\Delta(G) \geqslant 3$, 则 $\operatorname{lc}(G) \leqslant\left\lceil\frac{\Delta(G)}{2}\right\rceil+1$;

(2) 如果 $g(G) \geqslant 10$, 则 $\operatorname{lc}(G) \leqslant\left\lceil\frac{\Delta(G)}{2}\right\rceil+2$;

(3) 如果 $g(G) \geqslant 8$, 则 $\operatorname{lc}(G) \leqslant\left\lceil\frac{\Delta(G)}{2}\right\rceil+3$.

易见, 对于任意的图 $G$ 有 $\operatorname{lc}(G) \geqslant\left\lceil\frac{\Delta(G)}{2}\right\rceil+1$. 因此, 我们很自然的就要去考虑这样一个 问题: 哪些图可以达到这个下界呢? Raspaud 和 Wang ${ }^{[4]}$ 证明了下面结果:

定理 2 对于一个平面图 $G$, 若存在一个有序对 $(\Delta, g) \in\{(13,7),(7,9),(5,11),(3,13)\}$, 使得 $\Delta(G) \geqslant \Delta$ 和 $g(G) \geqslant g$, 则 $\mathrm{lc}(G)=\left\lceil\frac{\Delta(G)}{2}\right\rceil+1$.

本文考虑非负特征图的线性染色. Euclid 平面, 射影平面, 圆环面, Klein 瓶都是具有非 负 Euler 特征的曲面. 在整篇文章中我们将非负特征图简写为 $\mathrm{NC}$-图. 本文的主要结果如下:

定理 3 对于每一 NC-图 $G$, 若存在一个有序对 $(\Delta, g) \in\{(13,7),(9,8),(7,9),(5,10)$, $(3,13)\}$, 使得 $\Delta(G) \geqslant \Delta$ 且 $g(G) \geqslant g$, 则 $\operatorname{lc}(G)=\left\lceil\frac{\Delta(G)}{2}\right\rceil+1$.

因为平面图是非负特征图的一个子类, 所以定理 3 是定理 2 的一个推广和扩充.

对于一个非负特征图 $G$, 用 $F(G)$ 表示它的面集合. 任意的 $f \in F(G)$, 用 $B(f)$ 来表示 $f$ 的边界. 如果 $u_{1}, u_{2}, \ldots, u_{n}$ 是 $B(f)$ 上按顺时针方向的点, 则记为 $f=\left[u_{1} u_{2} \cdots u_{n}\right]$, 注意 到点的重复出现是允许的. 面的度是指它的边界上边的条数, 其中割边被计算两次. 对于任 意的 $x \in V(G) \cup F(G)$, 用 $d_{G}(x)$ 表示 $G$ 中 $x$ 的度. 在不产生混淆的情形下, 可以简单的用 $d(x)$ 代替 $d_{G}(x)$. 一个度为 $k$ 的顶点 (或面) 称为 $k$-点 (或 $k$-面), 我们用 $N_{G}(v)$ 来表示 $G$ 中 $v$ 的邻点的集合.

\section{2 主要结果}

设 $G$ 是一个 NC-图, $v \in V(G)$. 我们用 $n_{2}(v)$ 表示与 $v$ 相邻的 2-点的个数. 如果 $n_{2}(v)=d(v)$, 即 $v$ 的所有邻点为 2-点, 则称 $v$ 为坏点. 设 $v$ 是一个 2-点, 如果 $n_{2}(v) \geqslant 1$, 则 称 $v$ 为类型 $\mathrm{I}$; 如果 $v$ 正好和两个 3 -点相邻, 则称 $v$ 为类型 II.

设 $c$ 是 $G$ 的一个部分线性染色, 颜色集合为 $C$. 对于任意的 $v \in V(G)$, 用 $C_{2}(v)$ 来表示 $C$ 中正好在 $N_{G}(v)$ 上出现两次的颜色子集合. 这个记号将会在下面的讨论中多次用到.

为证明定理 3 , 我们证明下面一个更强的结果:

定理 4 设 $M$ 是一个正整数, $G$ 是一个NC-图, 使得 $\Delta(G) \leqslant M$. 如果 $G$ 满足下列条 件之一, 则 $\operatorname{lc}(G) \leqslant\left\lceil\frac{M}{2}\right\rceil+1$ :

(1) $M \geqslant 13$ 且 $g(G) \geqslant 7$,

(2) $M \geqslant 9$ 且 $g(G) \geqslant 8$,

(3) $M \geqslant 7$ 且 $g(G) \geqslant 9$,

(4) $M \geqslant 5$ 且 $g(G) \geqslant 10$,

(5) $M \geqslant 3$ 且 $g(G) \geqslant 13$.

证明 反证法. 假设定理不正确. 设 $G$ 是一个极小反例, 即 $G$ 是一个满足假设条件的 $\mathrm{NC}$-图, $\operatorname{lc}(G)>\left\lceil\frac{M}{2}\right\rceil+1$, 但对于任意的一个阶数更小的 NC-图 $H$ 满足和 $G$ 相同的 $M$ 和围 长条件, 则有 lc $(H) \leqslant\left\lceil\frac{M}{2}\right\rceil+1$. 容易看到 $G$ 是连通的. 接下来证明 $\delta(G) \geqslant 2$.

假设 $G$ 包含 1-点 $v$ 与 $u$ 相邻. 令 $H=G-v$, 则 $H$ 是一个 NC-图且满足 $\Delta(H) \leqslant \Delta(G)$ 和 $g(H)=g(G)$. 由 $G$ 的极小性, $H$ 有一个线性染色 $c$ 应用 $\left\lceil\frac{M}{2}\right\rceil+1$ 种颜色. 为将 $c$ 扩充到 
整个 $G$, 我们用不在 $C_{2}(u) \cup\{c(u)\}$ 中的颜色染 $v$. 因为

$$
\left|C_{2}(u)\right| \leqslant\left\lfloor\frac{d_{H}(u)}{2}\right\rfloor=\left\lfloor\frac{d_{G}(u)-1}{2}\right\rfloor \leqslant\left\lfloor\frac{\Delta(G)-1}{2}\right\rfloor \leqslant\left\lfloor\frac{M-1}{2}\right\rfloor=\left\lceil\frac{M}{2}\right\rceil-1,
$$

总有一种颜色可以染给 $v$. 但这与 $G$ 的选择矛盾!

在下面的证明中我们应用权转移的方法. 首先, 根据围长的要求选择一个合适的整数 $k>2$. 结合 NC-图上的 Euler 公式

$$
|V(G)|-|E(G)|+|F(G)| \geqslant 0
$$

和

可以得到下面的等式:

$$
\sum_{v \in V(G)} d(v)=\sum_{f \in F(G)} d(f)=2|E(G)|
$$

$$
\sum_{v \in V(G)}[(k-2) d(v)-2 k]+\sum_{f \in F(G)}(2 d(f)-2 k) \leqslant 0 .
$$

在 $V(G) \cup F(G)$ 上定义权函数 $w$, 对于 $v \in V(G)$ 令 $w(v)=(k-2) d(v)-2 k$, 对于 $f \in F(G)$ 令 $w(f)=2 d(f)-2 k$. 总的权和是非正数. 然后我们定义一系列的权转移规则并 且在所有的点和面上执行它. 一旦权转移结束, 就会产生一个新的权转移函数 $w^{\prime}$. 在整个权 转移过程中权和都是不变的, 但是 $w^{\prime}$ 满足下面性质 (I) 和 (II):

(I) 对于所有的 $x \in V(G) \cup F(G)$, 有 $w^{\prime}(x) \geqslant 0$;

(II) 存在某个 $x^{*} \in V(G) \cup F(G)$, 使得 $w\left(x^{*}\right)>0$.

这就导致了下面一个很明显的矛盾, 因此这样的反例是不存在的:

$$
0<\sum_{x \in V(G) \cup F(G)} w^{\prime}(x)=\sum_{x \in V(G) \cup F(G)} w(x) \leqslant 0 .
$$

在下面的的证明中, 我们简单记 $K=\left\lceil\frac{M}{2}\right\rceil+1$, 用 $C=\{1,2, \ldots, K\}$ 表示 $K$ 种颜色的集 合. 按照 $M$ 和围长的假设, 整个证明分为 5 个部分. 在每一部分中, 我们假设存在一个反例, 然后通过证明一系列的断言得到矛盾.

断言 $\mathbf{A}$ 假设 $|C|=K=\left\lceil\frac{M}{2}\right\rceil+1$. 设 $x$ 是一个 2 -点, $u$ 和 $v$ 为 $x$ 的邻点且 $d_{G}(v) \leqslant$ $d_{G}(u)$. 如果 $2 \leqslant d_{G}(v) \leqslant 4$, 则 $\left\lfloor\frac{d_{G}(u)-1}{2}\right\rfloor+d_{G}(v) \geqslant K$.

证明 假设 $\left\lfloor\frac{d_{G}(u)-1}{2}\right\rfloor+d_{G}(v) \leqslant K-1$. 设 $v_{1}, v_{2}, \ldots, v_{m}$ 是 $v$ 的不同于 $x$ 的邻点, 其 中 $m=d_{G}(v)-1$. 设 $H=G-x$, 则 $H$ 是一个 NC-图且满足 $|H|<|G|, \Delta(H) \leqslant \Delta(G) \leqslant M$, $g(H) \geqslant g(G)$. 由 $G$ 的极小性可知, $H$ 有一个线性 $K$-染色 $c$ 应用颜色集合 $C$. 我们给 $x$ 定 义一个可用的颜色列表如下:

$$
L(x)= \begin{cases}C \backslash\left(C_{2}(u) \cup\left\{c(u), c\left(v_{1}\right), \ldots, c\left(v_{m}\right)\right\}\right), & \text { 若 } c(u)=c(v), \\ C \backslash\left(C_{2}(u) \cup\left\{c(u), c(v), c\left(v_{1}\right), \ldots, c\left(v_{m-1}\right)\right\}\right), & \text { 若 } c(u) \neq c(v) .\end{cases}
$$

因为

$$
\left|C_{2}(u)\right| \leqslant\left\lfloor\frac{d_{H}(u)}{2}\right\rfloor=\left\lfloor\frac{d_{G}(u)-1}{2}\right\rfloor \leqslant K-1-d_{G}(v)
$$

故可以得到

$$
|L(x)| \geqslant|C|-\left|C_{2}(u)\right|-m-1 \geqslant K-\left(K-1-d_{G}(v)\right)-d_{G}(v)=1 .
$$

因此, 可用 $L(x)$ 中的一个颜色染 $x$, 从而将 $c$ 扩充到整个 $G$, 矛盾于 $G$ 的选择. 断言 $\mathrm{A}$ 证毕. 
第 1 部分 $M \geqslant 13$ 且 $g(G) \geqslant 7$, 则 $|C|=K=\left\lceil\frac{M}{2}\right\rceil+1 \geqslant 8$.

由断言 $\mathrm{A}$ 有

断言 1.1 设一个 2 -点 $x$ 与两个点 $v$ 和 $u$ 相邻且 $d(v) \leqslant d(u)$. 如果 $2 \leqslant d(v) \leqslant 4$, 则 $\left\lfloor\frac{d(u)-1}{2}\right\rfloor+d(v) \geqslant 8$.

从断言 1.1 即可得到下面的推论:

观察 1.1 设一个 2 -点 $x$ 和两个点 $v$ 和 $u$ 相邻且 $d(v) \leqslant d(u)$.

(1) 如果 $d(v)=2$, 则 $d(u) \geqslant 13$;

(2) 如果 $d(v)=3$, 则 $d(u) \geqslant 11$;

(3) 如果 $d(v)=4$, 则 $d(u) \geqslant 9$.

令等式 $(*)$ 中 $k=7$. 定义一个初始权函数: 如果 $v \in V(G)$, 则 $w(v)=5 d(v)-14$, 如果 $f \in F(G)$, 则 $w(f)=2 d(f)-14$. 在给出权转移规则之前引进一个概念.

设点 $v$ 满足 $d(v) \geqslant 14$ 或 $d(v)=13$ 且 $n_{2}(v)<13$. 如果存在 $G$ 中的一条路 $x x^{\prime} v^{\prime} v$ 满足 $d\left(x^{\prime}\right)=d\left(v^{\prime}\right)=2$, 则称 $x$ 为 $v$ 的悬挂点. 设 $p_{13}(v)$ 表示 $v$ 的悬挂坏 13-点的个数.

权转移规则如下:

(R1.1) 设 $d(v) \geqslant 14$.

如果 $d(v) \geqslant 15$ 或 $d(v)=14$ 且 $n_{2}(v)<d(v), v$ 转 4 给相邻 2-点, $\left(w(v)-4 n_{2}(v)-\frac{1}{2}\right) / p_{13}(v)$ 给每一个悬挂坏 13 -点 (若 $p_{13}(v)>0$ ).

如果 $d(v)=n_{2}(v)=14, v$ 转 4 给相邻的 2-点.

$(\mathrm{R} 1.2)$ 设 $d(v)=13$.

如果 $v$ 是坏点, 则 $v$ 转 4 给相邻的类型 I 2-点, 转 $\frac{15}{4}$ 给相邻的其他 2-点.

如果 $v$ 不是坏点, 则 $v$ 转 4 给相邻 2-点, $\left(w(v)-4 n_{2}(v)-\frac{1}{2}\right) / p_{13}(v)$ 给每一个悬挂坏 13-点 (若 $p_{13}(v)>0$ ).

(R1.3) 如果 $4 \leqslant d(v) \leqslant 12$, 则 $v$ 转 $\left(w(v)-\frac{1}{2}\right) / d(v)$ 给每一个相邻 2-点.

(R1.4) 每一个 3 -点转 $\frac{131}{396}$ 给每一个相邻的 2 -点.

(R1.5) 每一个 $d(f) \geqslant 8$ 的面 $f$ 转 1 给 $B(f)$ 中每一个相邻于两个类型 I 2-点的坏 13-点. 很容易从 (R1.1) 到 (R1.4) 得到下面的观察:

观察 1.2 设 $v$ 是一个度数不小于 3 的点, $u$ 是与 $v$ 相邻的一个 2 -点.

(1) 如果 $d(v) \geqslant 13$, 则 $v$ 给 $u$ 至少 $\frac{15}{4}$;

(2) 如果 $11 \leqslant d(v) \leqslant 12$, 则 $v$ 给 $u$ 至少 $\frac{81}{22}$;

(3) 如果 $d(v) \geqslant 9$, 则 $v$ 给 $u$ 至少 $\frac{61}{18}$;

(4) 如果 $d(v) \geqslant 5$, 则 $v$ 给 $u$ 至少 $\frac{21}{10}$;

(5) 如果 $d(v)=4$, 则 $v$ 给 $u$ 至少 $\frac{11}{8}$.

设 $w^{\prime}$ 为权转移结束后的新权函数. 我们来验证对于所有的 $x \in V(G) \cup F(G)$ 有 $w^{\prime}(x) \geqslant 0$, 且存在某个 $x^{*} \in V(G) \cup F(G)$, 使得 $w^{\prime}\left(x^{*}\right)>0$.

设 $f \in F(G)$. 因为 $g(G) \geqslant 7$, 所以 $d(f) \geqslant 7$. 如果 $d(f)=7$, 则易得

$$
w^{\prime}(f)=w(f)=0 .
$$

如果 $d(f)=8$, 则 $f$ 最多关联两个与 $B(f)$ 上两个类型 I 2-点相邻的坏 13-点. 因此, 由 
(R1.5) 可知

$$
w^{\prime}(f) \geqslant w(f)-2 \times 1=2 \times 8-14-2=0 .
$$

如果 $d(f) \geqslant 9$, 则 $f$ 最多关联 $\left\lfloor\frac{d(f)}{3}\right\rfloor$ 个与 $B(f)$ 上两个类型 I 2-点相邻的坏 13-点. 因 此, 由 (R1.5) 可知

$$
w^{\prime}(f) \geqslant w(f)-\left\lfloor\frac{d(f)}{3}\right\rfloor \geqslant 2 d(f)-14-\frac{1}{3} d(f)=\frac{5}{3} d(f)-14 \geqslant 1 .
$$

设 $v \in V(G)$. 因为 $\delta(G) \geqslant 2$, 所以 $d(v) \geqslant 2$.

假设 $d(v) \geqslant 14$. 如果 $d(v) \geqslant 15$ 或 $d(v)=14$ 且 $n_{2}(v)<d(v)$, 则

$$
w(v)-4 n_{2}(v)=5 d(v)-14-4 n_{2}(v)=4\left(d(v)-n_{2}(v)\right)+(d(v)-14) \geqslant 1,
$$

由 (R1.1) 可知

$$
w^{\prime}(v)=w(v)-4 n_{2}(v)-p_{13}(v) \times \frac{\left(w(v)-4 n_{2}(v)-\frac{1}{2}\right)}{p_{13}(v)}=\frac{1}{2} .
$$

如果 $d(v)=n_{2}(v)=14$, 也就是说, $v$ 是坏 14-点, 则由 (R1.1) 可知

$$
w^{\prime}(v)=w(v)-4 n_{2}(v)=d(v)-14=0 .
$$

如果 $4 \leqslant d(v) \leqslant 12$, 则 $w(v)=5 d(v)-14 \geqslant 6$. 因此, 由 (R1.3) 可知

$$
w^{\prime}(v)=w(v)-n_{2}(v) \times \frac{\left(w(v)-\frac{1}{2}\right)}{n_{2}(v)}=\frac{1}{2} .
$$

如果 $d(v)=3$, 则 $w(v)=5 d(v)-14=1$. 由 (R1.4) 可知 $v$ 转 $\frac{131}{396}$ 给相邻的 2-点. 所以,

$$
w^{\prime}(v) \geqslant 1-3 \times \frac{131}{396}=\frac{1}{132} \text {. }
$$

假设 $d(v)=2$, 则 $w(v)=5 d(v)-14=-4$. 设 $x$ 和 $y$ 是 $v$ 的邻点且 $d(x) \leqslant d(y)$. 首先， 假设 $v$ 不是类型 I 2-点, 则 $d(x) \geqslant 3$. 如果 $d(x)=3$, 则由观察 1.1 和 1.2 可知, $d(y) \geqslant 11$ 且

$$
w^{\prime}(v) \geqslant-4+\frac{131}{396}+\frac{81}{22}=\frac{5}{396} .
$$

如果 $d(x)=4$, 则由观察 1.1 和 1.2 可知, $d(y) \geqslant 9$ 且

$$
w^{\prime}(v) \geqslant-4+\frac{11}{8}+\frac{61}{18}=\frac{55}{72} \text {. }
$$

如果 $d(x) \geqslant 5$, 则由观察 1.2(4) 可知

$$
w^{\prime}(v)=-4+2 \times \frac{21}{10}=\frac{1}{5} .
$$

接下来假设 $v$ 是类型 I 2-点, 即 $d(x)=2$. 由观察 $1.1, d(y) \geqslant 13$. 如果 $d(y) \geqslant 14$, 则由 (R1.1), $y$ 转 4 给 $v$. 因此, $w^{\prime}(v)=-4+4=0$. 如果 $d(y)=13$, 则由 (R1.2), $y$ 转 4 给 $v$. 因 此, $w^{\prime}(v)=-4+4=0$.

假设 $d(v)=13$, 则 $w(v)=5 d(v)-14=51$. 如果 $v$ 最多和 12 个 2 -点相邻, 则 $51-4 n_{2}(v) \geqslant$ $51-4 \times 12=3$, 所以由 (R1.2) 可知

$$
w^{\prime}(v)=w(v)-4 n_{2}(v)-p_{13}(v) \times \frac{\left(w(v)-4 n_{2}(v)-\frac{1}{2}\right)}{p_{13}(v)}=\frac{1}{2} .
$$

因此, 假设 $n_{2}(v)=13$, 即 $v$ 为坏 13-点. 如果 $v$ 至多和 9 个类型 I 2-点相邻, 则由 (R1.2) 可知

$$
w^{\prime}(v) \geqslant 51-4 \times 9-\frac{15}{4} \times 4=0 .
$$


因此, 进一步假设 $v$ 至少和 10 个类型 I 2-点相邻. 如果 $v$ 与某个面 $f^{*}$ 关联且 $d\left(f^{*}\right) \geqslant 8$, 使得 $B\left(f^{*}\right)$ 上两个类型 I 2-点与 $v$ 相邻, 则由 (R1.5), $f^{*}$ 转 1 给 $v$. 所以

$$
w^{\prime}(v) \geqslant 51+1-4 \times 13=0 .
$$

于是假设这样的面 $f^{*}$ 不存在. 设 $x_{1}, x_{2}, \ldots, x_{13}$ 为 $v$ 的按某个方向排列的邻点. 因为 $d(v)=13$ 且 $v$ 至多相邻 3 个非类型 I 2-点, 所以必然存在 4 个连续的类型 I 2-点, 设为 $x_{1}, x_{2}, x_{3}, x_{4}$. 设 $y_{i}, 1 \leqslant i \leqslant 4$, 为 $x_{i}$ 的不同于 $v$ 的邻点. 因为 $d\left(y_{i}\right)=2$, 设 $z_{i}$ 为 $y_{i}$ 的不同 于 $x_{i}$ 的邻点. 由观察 $1.1, d\left(z_{i}\right) \geqslant 13$. 对于 $1 \leqslant j \leqslant 3$, 设 $f_{j}$ 为边界包含边 $v x_{j}$ 和 $v x_{j+1}$ 的 面. 由前面的证明, $f_{j}$ 为 7-面, 也就是说, $f_{j}=\left[v x_{j} y_{j} z_{j} z_{j+1} y_{j+1} x_{j+1}\right]$. 因此, 对于 $2 \leqslant k \leqslant 3$, 因为 $z_{k}$ 和 $z_{k-1}, z_{k+1}$ 相邻, 所以每一个 $z_{k}$ 相邻至多 $d\left(z_{k}\right)-2$ 个 2-点. 即 $v$ 是 $z_{2}$ 和 $z_{3}$ 的悬 挂坏 13-点. 设 $\sigma\left(z_{k}\right)$ 为根据 (R1.1) 和 (R1.2) 从 $z_{k}$ 转给 $v$ 的总权和. 我们有下面的不等式:

$$
\begin{aligned}
\sigma\left(z_{k}\right) & \geqslant \frac{w\left(z_{k}\right)-4 n_{2}\left(z_{k}\right)-\frac{1}{2}}{p_{13}\left(z_{k}\right)} \\
& \geqslant \frac{5 d\left(z_{k}\right)-14-4\left(d\left(z_{k}\right)-2\right)-\frac{1}{2}}{d\left(z_{k}\right)-2} \\
& =1-\frac{4+\frac{1}{2}}{d\left(z_{k}\right)-2} \\
& \geqslant \frac{13}{22},
\end{aligned}
$$

因此,

$$
w^{\prime}(v) \geqslant 51+\sigma\left(z_{2}\right)+\sigma\left(z_{3}\right)-4 n_{2}(v) \geqslant 51+2 \times \frac{13}{22}-4 \times 13=\frac{2}{11} .
$$

到现在为止我们证明了 $w^{\prime}$ 满足性质 (I).

假设 $w^{\prime}$ 不满足性质 (II), 也就是说, 对于所有的 $x \in V(G) \cup F(G)$, 有 $w^{\prime}(x)=0$ 成立, 则 根据先前的证明可以看到: 对于所有的 $f \in F(G)$, 必有 $d(f) \in\{7,8\}$; 对于所有的 $v \in V(G)$, 必有 $d(v) \in\{2,13,14\}$. 此外, 所有的 2-点都是类型 I 2-点, 所有的 13-点和 14-点都是坏点. 这就暗含了每一个点 $v$, 满足 $13 \leqslant d(v) \leqslant 14$, 相邻 $d(v)$ 个类型 I 2 -点.

显然, $G$ 包含一个面 $f=\left[v_{1} v_{2} \cdots v_{m}\right](7 \leqslant m \leqslant 8)$. 由观察 $1.1, G$ 不包含 3 个连续相邻 2 -点, 所以必然存在边界上一点 $v_{1}$, 使得 $13 \leqslant d\left(v_{1}\right) \leqslant 14$. 因此, 对于所有的 $i=2,3, m-1, m$ 有 $d\left(v_{i}\right)=2$, 且对于 $j=4, m-2$ 有 $d\left(v_{j}\right) \geqslant 13$. 如果 $m=7$, 因为 $v_{4}$ 和 $v_{5}$ 都和一个度数大 于 2 的点相邻, 所以他们不是坏点. 如果 $m=8$, 因为 $v_{4}$ 和 $v_{6}$ 都是坏点, 因此 $v_{5}$ 不是类型 I 2-点. 这与前面的事实矛盾, 所以性质 (II) 是满足的. 证毕.

第 2 部分 $M \geqslant 9$ 且 $g(G) \geqslant 8$, 则 $|C|=K=\left\lceil\frac{M}{2}\right\rceil+1 \geqslant 6$.

由断言 $\mathrm{A}$, 有

断言 2.1 设一个 2 -点 $x$ 和两个点 $v$ 和 $u$ 相邻且 $d(v) \leqslant d(u)$. 如果 $2 \leqslant d(v) \leqslant 4$, 则 $\left\lfloor\frac{d(u)-1}{2}\right\rfloor+d(v) \geqslant 6$.

从断言 2.1 立即可以得到下面一个推论:

观察 2.1 设一个 2 -点 $x$ 和两个点 $v, u$ 相邻且 $d(v) \leqslant d(u)$.

(1) 如果 $d(v)=2$, 则 $d(u) \geqslant 9$;

(2) 如果 $d(v)=3$, 则 $d(u) \geqslant 7$;

(3) 如果 $d(v)=4$, 则 $d(u) \geqslant 5$. 
令等式 $(*)$ 中 $k=8$. 定义一个初始权函数 $w$ : 如果 $v \in V(G)$, 则 $w(v)=6 d(v)-16$; 如 果 $f \in F(G)$, 则 $w(f)=2 d(f)-16$.

权转移规则如下:

(R2) 设 $v$ 是一个度至少 3 的点, $u$ 是相邻于 $v$ 的一个 2-点.

如果 $d(v) \geqslant 9$, 则 $v$ 给 $u$ 的权为 $\frac{37}{9}$;

如果 $d(v)=7,8$, 则 $v$ 给 $u$ 的权为 $\frac{25}{7}$;

如果 $d(v)=5,6$, 则 $v$ 给 $u$ 的权为 $\frac{13}{5}$;

如果 $d(v)=4$, 则 $v$ 给 $u$ 的权为 $\frac{8}{5}$;

如果 $d(v)=3$, 则 $v$ 给 $u$ 的权为 $\frac{4}{7}$.

设 $w^{\prime}$ 为权转移结束后的新权函数. 设 $f \in F(G)$. 因为 $g(G) \geqslant 8$, 所以 $d(f) \geqslant 8$, 则

$$
w^{\prime}(f)=w(f)=2 d(f)-16 \geqslant 0 .
$$

设 $v \in V(G)$. 因为 $\delta(G) \geqslant 2$, 所以 $d(v) \geqslant 2$. 如果 $d(v) \geqslant 9$, 则由 $(\mathrm{R} 2), v$ 转 $\frac{37}{9}$ 给相邻 的 2 -点, 所以

$$
w^{\prime}(v) \geqslant w(v)-\frac{37}{9} d(v)=6 d(v)-16-\frac{37}{9} d(v)=\frac{17}{9} d(v)-16 \geqslant 1 .
$$

如果 $7 \leqslant d(v) \leqslant 8$, 则由 (R2), $v$ 转 $\frac{25}{7}$ 给相邻的 2 -点, 所以

$$
w^{\prime}(v) \geqslant w(v)-\frac{25}{7} d(v)=6 d(v)-16-\frac{25}{7} d(v)=\frac{17}{7} d(v)-16 \geqslant 1 .
$$

如果 $5 \leqslant d(v) \leqslant 6$, 则由 (R2), $v$ 转 $\frac{13}{5}$ 给相邻的 2 -点, 所以

$$
w^{\prime}(v) \geqslant w(v)-\frac{13}{5} d(v)=6 d(v)-16-\frac{13}{5} d(v)=\frac{17}{5} d(v)-16 \geqslant 1 .
$$

如果 $d(v)=4$, 则由 $(\mathrm{R} 2), w(v)=6 d(v)-16=8$ 且

如果 $d(v)=3$, 则由 $(\mathrm{R} 2)$,

$$
w^{\prime}(v) \geqslant 8-4 \times \frac{8}{5}=\frac{8}{5} \text {. }
$$

$$
w^{\prime}(v)=2-3 \times \frac{4}{7}=\frac{2}{7} .
$$

假设 $d(v)=2$, 则 $w(v)=6 d(v)-16=-4$. 设 $x$ 和 $y$ 为 $v$ 的邻点且满足 $d(x) \leqslant d(y)$. 如 果 $d(y) \geqslant 9$, 则由 (R2), $y$ 转 $\frac{37}{9}$ 给 $v$, 所以

$$
w^{\prime}(v) \geqslant-4+\frac{37}{9}=\frac{1}{9} \text {. }
$$

假设 $d(y) \leqslant 8$. 由观察 $2.1, d(x) \geqslant 3$ 且 $d(y) \geqslant 5$. 如果 $d(x) \geqslant 5$, 则由 (R2), $x, y$ 分别转 $\frac{13}{5}$ 给 $v$, 所以

$$
w^{\prime}(v) \geqslant-4+2 \times \frac{13}{5}=\frac{6}{5} .
$$

如果 $d(x)=4$, 则由 $(\mathrm{R} 2)$,

$$
w^{\prime}(v) \geqslant-4+\frac{8}{5}+\frac{13}{5}=\frac{1}{5} .
$$

如果 $d(x)=3$, 则由观察 2.1 和 (R2) 可知, $d(y) \geqslant 7$ 且

$$
w^{\prime}(v) \geqslant-4+\frac{4}{7}+\frac{25}{7}=\frac{1}{7} .
$$

至此, 我们证明了对所有的 $x \in V(G)$, 有 $w^{\prime}(x)>0$, 且对所有的 $x \in F(G)$, 有 $w^{\prime}(x) \geqslant 0$. 因此, (I) 和 (II) 成立. 证毕.

第 3 部分 $\quad M \geqslant 7$ 且 $g(G) \geqslant 9$, 则 $|C|=K=\left\lceil\frac{M}{2}\right\rceil+1 \geqslant 5$. 
由断言 $\mathrm{A}$, 有

断言 3.1 设一个 2 -点 $x$ 和两个点 $v$ 和 $u$ 相邻且 $d(v) \leqslant d(u)$. 如果 $2 \leqslant d(v) \leqslant 3$, 则 $\left\lfloor\frac{d(u)-1}{2}\right\rfloor+d(v) \geqslant 5$.

观察 3.1 设一个 2 -点 $x$ 和两个点 $v$ 和 $u$ 相邻且 $d(v) \leqslant d(u)$.

(1) 如果 $d(v)=2$, 则 $d(u) \geqslant 7$.

(2) 如果 $d(v)=3$, 则 $d(u) \geqslant 5$.

令等式 $(*)$ 中 $k=9$. 定义一个初始权函数 $w$ : 如果 $v \in V(G)$, 则 $w(v)=7 d(v)-18$; 如 果 $f \in F(G)$, 则 $w(f)=2 d(f)-18$.

权转移规则如下:

(R3) 设 $v$ 是一个度至少 3 的点, $u$ 是相邻于 $v$ 一个 2 -点.

如果 $d(v) \geqslant 7$, 则 $v$ 给 $u$ 的权为 $\frac{61}{14}$;

如果 $d(v)=6$, 则 $v$ 给 $u$ 的权为 $\frac{47}{12}$;

如果 $d(v)=5$, 则 $v$ 给 $u$ 的权为 $\frac{33}{10}$;

如果 $d(v)=4$, 则 $v$ 给 $u$ 的权为 $\frac{19}{8}$;

如果 $d(v)=3$, 则 $v$ 给 $u$ 的权为 $\frac{5}{6}$.

设 $w^{\prime}$ 为权转移结束后的新权函数. 只需验证对于所有的 $x \in V(G) \cup F(G)$ 有 $w^{\prime}(x) \geqslant 0$, 且存在某个 $x^{*} \in V(G) \cup F(G)$, 使得 $w^{\prime}\left(x^{*}\right)>0$.

设 $f \in F(G)$. 因为 $g(G) \geqslant 9$, 所以 $d(f) \geqslant 9$, 则 $w^{\prime}(f)=w(f)=2 d(f)-18 \geqslant 0$.

设 $v \in V(G)$, 则 $d(v) \geqslant 2$. 如果 $d(v) \geqslant 7$, 则由 (R3),

$$
w^{\prime}(v)=w(v)-\frac{61}{14} n_{2}(v) \geqslant 7 d(v)-18-\frac{61}{14} d(v)=\frac{37}{14} d(v)-18 \geqslant \frac{37}{14} \times 7-18=\frac{1}{2} .
$$

如果 $d(v)=6$, 则由 (R3),

如果 $d(v)=5$, 则由 (R3),

$$
w^{\prime}(v) \geqslant 24-\frac{47}{12} \times 6=\frac{1}{2}
$$

如果 $d(v)=4$, 则由 (R3),

$$
w^{\prime}(v) \geqslant 17-\frac{33}{10} \times 5=\frac{1}{2}
$$

如果 $d(v)=3$, 则由 (R3),

$$
w^{\prime}(v) \geqslant 10-\frac{19}{8} \times 4=\frac{1}{2}
$$

$$
w^{\prime}(v) \geqslant 3-\frac{5}{6} \times 3=\frac{1}{2} .
$$

假设 $d(v)=2$, 则 $w(v)=7 d(v)-18=-4$. 设 $x$ 和 $y$ 为 $v$ 的邻点且满足 $d(x) \leqslant d(y)$. 如 果 $d(y) \geqslant 7$, 则由 (R3), $y$ 转 $\frac{61}{14}$ 给 $v$. 所以,

$$
w^{\prime}(v) \geqslant-4+\frac{61}{14}=\frac{5}{14} \text {. }
$$

假设 $d(v) \leqslant 6$. 由观察 $3.1, d(x) \geqslant 3$. 如果 $d(x) \geqslant 4$, 由 (R3), $x$ 和 $y$ 分别转 $\frac{19}{8}$ 给 $v$, 所 以

$$
w^{\prime}(v) \geqslant-4+2 \times \frac{19}{8}=\frac{3}{4} .
$$

如果 $d(x)=3$, 由观察 $3.1, d(y) \geqslant 5$, 所以

$$
w^{\prime}(v) \geqslant-4+\frac{33}{10}+\frac{5}{6}=\frac{2}{15} \text {. }
$$


证毕.

第 4 部分 $\quad M \geqslant 5$ 且 $g(G) \geqslant 10$.

在这种情形下, $|C|=K=\left\lceil\frac{M}{2}\right\rceil+1 \geqslant 4$. 如果 $M \geqslant 7$, 则由第 3 部分可知结果成立. 因 此假设 $5 \leqslant M \leqslant 6$, 即 $|C|=4$ 且 $\Delta(G) \leqslant 6$.

断言 $\mathrm{A}$ 隐含着下面的断言 4.1 :

断言 4.1 设 $x u v y$ 是一条路且满足 $d(u)=d(v)=2$, 则 $d(x) \geqslant 5, d(y) \geqslant 5$.

断言 4.2 若一个 3 -点 $u$ 相邻于 3 个 2 -点, 则每个 2 -点都相邻一个度至少为 4 的点.

证明 假设断言不正确. 设 $u$ 相邻 3 个 2 -点 $x, y, z$, 设 $x^{\prime}, y^{\prime}, z^{\prime}$ 分别为 $x, y, z$ 的不同 于 $u$ 的邻点. 由断言 $4.1, x^{\prime}, y^{\prime}, z^{\prime}$ 的度数至 少为 3 .

假设 $d\left(x^{\prime}\right)=3$. 设 $s_{1}$ 和 $s_{2}$ 为 $x^{\prime}$ 的不同 于 $x$ 的邻点, 如图 1 所示. 令 $H=G-x$, 则 $H$ 是一个 NC-图且满足 $\Delta(G) \leqslant \Delta(H) \leqslant M$ 和 $g(H) \geqslant g(G) \geqslant 10$. 由 $G$ 的极小性, $H$ 有一个 线性 $K$-染色 $c$ 应用颜色集合 $C=\{1,2,3,4\}$.

考虑下面两种情形:

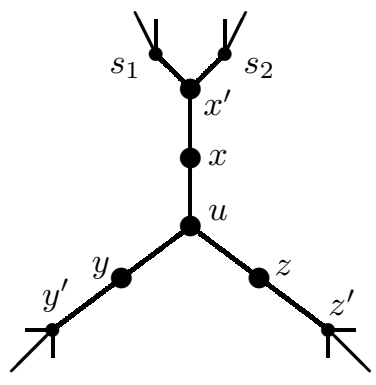

图 1 断言 4.2 中的结构

情形 $1 \quad c(u)=c\left(x^{\prime}\right)$.

如果 $c\left(s_{1}\right)=c\left(s_{2}\right)$, 我们用 $C \backslash\left\{c(u), c\left(s_{1}\right)\right\}$ 中在 $y$ 和 $z$ 上最多出现 1 次的颜色染 $x$. 如 果 $c\left(s_{1}\right) \neq c\left(s_{2}\right)$, 则用不同于 $c(u), c(y), c(z)$ 的颜色染 $x$. 因为 $|C|=4$, 这样的颜色总是存在 的.

情形 $2 \quad c(u) \neq c\left(x^{\prime}\right)$.

如果存在一个颜色 $a \in C \backslash\left\{c(u), c\left(x^{\prime}\right)\right\}$ 至多在 $s_{1}$ 和 $s_{2}$ 上出现 1 次, 同时至多在 $y$ 和 $z$ 上出现一次, 则给 $x$ 染 $a$. 否则, 不失一般性, 假设 $c\left(x^{\prime}\right)=1, c(u)=2, c\left(s_{1}\right)=c\left(s_{2}\right)=3$, $c(y)=c(z)=4$. 首先, 给 $x$ 染 2 . 然后, 如果 $c\left(y^{\prime}\right)=c\left(z^{\prime}\right)=1$, 给 $u$ 染 3 ; 否则, 给 $u$ 染 1 .

在上面的每一种情形, 都可以 得到 $G$ 的一个线性 $K$-染色. 这与 $G$ 的选取矛盾. 因此, $d\left(x^{\prime}\right) \geqslant 4$. 同 理可证, $d\left(y^{\prime}\right) \geqslant 4, d\left(z^{\prime}\right) \geqslant 4$. 断言 证毕.

断言 4.3 不存在两条路 $u_{1} u_{2} \cdots u_{5}$ 和 $v_{1} v_{2} v_{3}$, 使得 $d\left(u_{1}\right)=$ $d\left(u_{3}\right)=d\left(v_{2}\right)=3, d\left(u_{2}\right)=d\left(u_{4}\right)=$

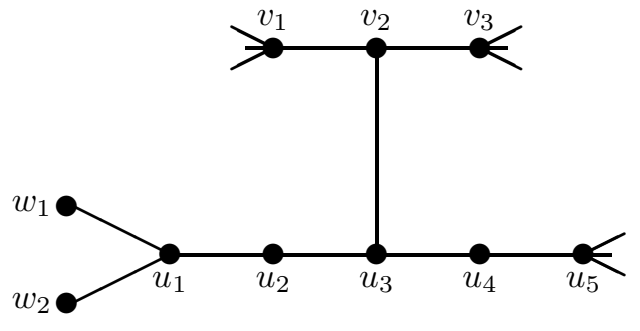

图 2 断言 4.3 中的子结构 2 , 且 $u_{3}$ 和 $v_{2}$ 相邻.

证明 假设 $G$ 包含这样两条路, 如图 2 所示. 设 $H=G-u_{2}$. 则 $H$ 是一个 NC-图且满 足 $\Delta(H) \leqslant \Delta(G)$ 和 $g(H) \geqslant g(G) \geqslant 10$. 由 $G$ 的极小性, $H$ 有一个线性 $K$-染色 $c$ 应用颜色 集合 $C=\{1,2,3,4\}$. 不失一般性, 考虑下面两种情形:

情形 $1 \quad c\left(u_{4}\right) \neq c\left(v_{2}\right)$. 
设 $w_{1}$ 和 $w_{2}\left(\neq u_{2}\right)$ 为 $u_{1}$ 的邻点. 如果 $c\left(u_{1}\right) \neq c\left(u_{3}\right)$, 我们用 $C \backslash\left\{c\left(u_{1}\right), c\left(u_{3}\right), c\left(w_{1}\right)\right\}$ 中的颜色染 $u_{2}$. 如果 $c\left(u_{1}\right)=c\left(u_{3}\right)$, 则用 $C \backslash\left\{c\left(u_{1}\right), c\left(w_{1}\right), c\left(w_{2}\right)\right\}$ 中的颜色染 $u_{2}$.

情形 $2 \quad c\left(u_{4}\right)=c\left(v_{2}\right)=1$.

如果 $c\left(u_{1}\right)=c\left(u_{3}\right)$, 我们用 $C \backslash\left\{1, c\left(u_{1}\right), c\left(w_{1}\right)\right\}$ 中的颜色染 $u_{2}$. 假设 $c\left(u_{1}\right) \neq c\left(u_{3}\right)$, 设 $c\left(u_{1}\right)=2, c\left(u_{3}\right)=3$. 如果 4 在集合 $\left\{c\left(w_{1}\right), c\left(w_{2}\right)\right\}$ 中至多出现 1 次, 则给 $u_{2}$ 染 4 . 因此, 假 设 $c\left(w_{1}\right)=c\left(w_{2}\right)=4$. 容易看到存在一个颜色 $a \in\{2,4\}$ 在集合 $\left\{c\left(v_{1}\right), c\left(v_{3}\right), c\left(u_{5}\right)\right\}$ 中最多 出现 1 次. 我们给 $u_{2}$ 染 3 , 然后改染 $u_{3}$ 为 $a$. 断言证毕.

令等式 $(*)$ 中 $k=10$. 定义一个初始权函数 $w$ : 如果 $v \in V(G)$, 则 $w(v)=8 d(v)-20$; 如 果 $f \in F(G)$, 则 $w(f)=2 d(f)-20$.

权转移规则如下:

(R4.1) 每一个度数至少是 6 的点转 $\frac{55}{12}$ 给相邻 2 -点.

(R4.2) 每一个 5 -点转 4 给相邻 2 -点.

(R4.3) 每一个 4 -点转 $\frac{23}{8}$ 给相邻 2 -点.

(R4.4) 设 3-点 $v$ 和 2-点 $u$ 相邻. 如果 $u$ 相邻两个 3 -点, 则 $v$ 给 $u$ 染 2 ; 否则, $v$ 给 $u$ 染 $\frac{4}{3}$

设 $w^{\prime}$ 为权转移结束后的新权函数. 只需验证 $w^{\prime}$ 满足 (I) 和 (II).

设 $f \in F(G)$. 因为 $g(G) \geqslant 10$, 所以 $d(f) \geqslant 10$. 如果 $d(f)=10$, 则 $w^{\prime}(f)=w(f)=0$. 如 果 $d(f) \geqslant 11$, 则 $w^{\prime}(f)=w(f)=2 d(f)-20 \geqslant 2$.

设 $v \in V(G)$. 因为 $\delta(G) \geqslant 2$, 所以 $d(v) \geqslant 2$. 如果 $d(v) \geqslant 6$, 则由 (R4.1),

$$
w^{\prime}(v) \geqslant w(v)-\frac{55}{12} d(v)=8 d(v)-20-\frac{55}{12} d(v)=\frac{41}{12} d(v)-20 \geqslant \frac{1}{2} .
$$

如果 $d(v)=5$, 则由 $(\mathrm{R} 4.2)$,

$$
w^{\prime}(v) \geqslant w(v)-4 \times 5=8 d(v)-20-20=0 .
$$

如果 $d(v)=4$, 则由 $(\mathrm{R} 4.3)$,

$$
w^{\prime}(v) \geqslant w(v)-4 \times \frac{23}{8}=\frac{1}{2} .
$$

假设 $d(v)=3$, 则 $w(v)=8 d(v)-20=4$. 如果 $v$ 至多和两个 2-点相邻, 则由 (R4.4), $w^{\prime}(v) \geqslant 4-2-2=0$. 否则, $n_{2}(v)=3$, 则由断言 4.2 , 每一个 2 -点都和一个度至少为 4 的点 相邻. 所以由 (R4.4),

$$
w^{\prime}(v) \geqslant 4-3 \times \frac{4}{3}=0 .
$$

假设 $d(v)=2$, 则 $w(v)=8 d(v)-20=-4$. 设 $x$ 和 $y$ 为 $v$ 的邻点且满足 $d(x) \leqslant d(y)$. 如 果 $d(y) \geqslant 6$, 则由 $(\mathrm{R} 4.1)$,

$$
w^{\prime}(v) \geqslant-4+\frac{55}{12}=\frac{7}{12} .
$$

如果 $d(y)=5$, 则由 $(\mathrm{R} 4.2)$,

$$
w^{\prime}(v) \geqslant-4+4=0 .
$$

如果 $d(y)=4$, 则由断言 $4.1, d(x) \geqslant 3$, 所以由转移规则可知,

$$
w^{\prime}(v) \geqslant-4+\frac{23}{8}+\frac{4}{3}=\frac{5}{24} \text {. }
$$

如果 $d(y)=3$, 则 $d(x)=3$, 由 $(\mathrm{R} 4.4), w^{\prime}(v)=-4+2+2=0$. 
到此我们证明了 $w^{\prime}$ 满足性质 (I).

假设 $w^{\prime}$ 不满足性质 $(\mathrm{II})$, 也就是说, 对于所有的 $x \in V(G) \cup F(G)$, 有 $w^{\prime}(x)=0$. 根据 前面讨论, 对所有 $f \in F(G)$, 有 $d(f)=10$; 对所有 $v \in V(G)$, 有 $d(v) \in\{2,3,5\}$, 并且每一个 5 -点和 5 个类型 I 2-点相邻, 即它为坏点.

假设 $G$ 包含一个 3 -点 $v$ 和 $v_{1}, v_{2}, v_{3}$ 相邻, 使得 $d\left(v_{1}\right) \leqslant d\left(v_{2}\right) \leqslant d\left(v_{3}\right)$. 因为所有的 5-点 都是坏点, 所以 $d\left(v_{3}\right) \leqslant 3$. 如果 $d\left(v_{3}\right)=2$, 则由断言 $4.2, v_{3}$ 的不同于 $v$ 的邻点度至少为 5 . 则由 (R4.2) 和 (R4.4),

$$
w^{\prime}\left(v_{3}\right) \geqslant-4+\frac{4}{3}+4=\frac{4}{3},
$$

因此可以得到 $d\left(v_{3}\right)=3$. 如果 $d\left(v_{2}\right)=3$, 则 $n_{2}(v) \leqslant 1$ 且由 $(\mathrm{R} 4.4), w^{\prime}(v) \geqslant 4-2=2$. 所 以 $d\left(v_{1}\right)=d\left(v_{2}\right)=2$. 设 $x \neq v$ 是 $v_{1}$ 的第 2 个邻点. 由断言 4.1 和 $4.3, d(x) \neq 2,3$, 所以 $d(x)=5$. 由 (R4.2) 和 (R4.3), $w^{\prime}\left(v_{1}\right) \geqslant-4+\frac{4}{3}+4=\frac{4}{3}$. 于是得到一个矛盾! 所以 $G$ 不包含 3 -点.

假设 $G$ 包含面 $f=\left[v_{1} v_{2} \cdots v_{10}\right]$. 由断言 $4.1, G$ 不包含 3 个连续相邻的 2-点, 所以必然 存在 $f$ 边界上某一点 $v_{1}$, 使得 $d\left(v_{1}\right)=5$. 因此, 对于所有的 $i=2,3,9,10$, 有 $d\left(v_{i}\right)=2$; 对于 $j=4,8$, 有 $d\left(v_{j}\right)=5$. 因为 $v_{4}$ 和 $v_{8}$ 都相邻 5 个类型 I 2-点, 所以对于所有的 $k=5,6,7$, 有 $d\left(v_{k}\right)=2$, 这与断言 4.1 矛盾! 因此, $w^{\prime}$ 满足 (II). 证毕.

第 5 部分 $M \geqslant 3$ 且 $g(G) \geqslant 13$.

现在, $|C|=K=\left\lceil\frac{M}{2}\right\rceil+1 \geqslant 3$. 如果 $M \geqslant 5$, 则由第 4 部分可知结果成立. 因此假设 $3 \leqslant M \leqslant 4$, 即 $|C|=3$ 且 $\Delta(G) \leqslant 4$.

断言 5.1 不存在一个 2 -点和两个 2 -点相邻.

证明 假设 $G$ 包含一个 2 -点 $v$ 和两个 2 -点 $x$ 和 $y$ 相邻. 设 $x^{\prime}$ 是 $x$ 的不同于 $v$ 的邻 点. 由 $G$ 的极小性, $G-v$ 有一个线性 3-染色 $v$. 如果 $c(x) \neq c(y)$, 用 $C \backslash\{c(x), c(y)\}$ 中的颜 色染 $v$. 如果 $c(x)=c(y)$, 则用 $C \backslash\left\{c(x), c\left(x^{\prime}\right)\right\}$ 中的颜色染 $v$. 这样就可以将 $c$ 扩充到整个 图 G. 矛盾! 断言证毕.

断言 5.2 不存在两条路 $x_{1} x_{2} \cdots x_{6}$ 和 $u_{1} u_{2} \cdots u_{7}$, 使得 $d\left(x_{2}\right)=d\left(x_{4}\right)=d\left(x_{5}\right)=$ $d\left(u_{2}\right)=d\left(u_{3}\right)=d\left(u_{5}\right)=d\left(u_{6}\right)=2, d\left(x_{3}\right)=d\left(u_{4}\right)=3$, 并且 $x_{3}$ 和 $u_{4}$ 相邻.

证明 假设 $G$ 包含着这样两条路, 如图 3 所示. 设 $H=G-u_{3}$, 则 $H$ 是一个 NC-图且 满足 $\Delta(H) \leqslant \Delta(G) \leqslant 4$ 和 $g(H) \geqslant g(G) \geqslant 13$. 由 $G$ 的极小性, $H$ 有一个线性 3-染色 $c$ 应用 颜色集合 $C=\{1,2,3\}$. 不失一般性, 考虑下面两种情形:

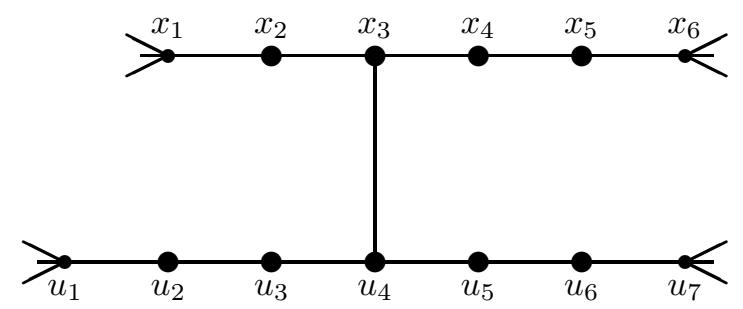

图 3 断言 5.2 中的子结构

情形 $1 \quad c\left(x_{3}\right) \neq c\left(u_{5}\right)$. 
如果 $c\left(u_{2}\right)=c\left(u_{4}\right)$, 我们用 $C \backslash\left\{c\left(u_{1}\right), c\left(u_{2}\right)\right\}$ 中的颜色染 $u_{3}$. 如果 $c\left(u_{2}\right) \neq c\left(u_{4}\right)$, 我们 用 $C \backslash\left\{c\left(u_{2}\right), c\left(u_{4}\right)\right\}$ 中的颜色染 $u_{3}$.

情形 $2 \quad c\left(x_{3}\right)=c\left(u_{5}\right)=1$.

不失一般性, 假设 $c\left(u_{4}\right)=2$.

子情形 $2.1 \quad c\left(u_{6}\right)=2$.

如果 $c\left(u_{2}\right) \in\{1,2\}$, 则给 $u_{3}$ 染 3 . 如果 $c\left(u_{2}\right)=3$, 则改染 $u_{5}$ 为 3 , 然后给 $u_{3}$ 染 1 .

子情形 $2.2 \quad c\left(u_{6}\right)=3$.

如果 $c\left(u_{2}\right) \in\{1,2\}$, 给 $u_{3}$ 染 3 . 假设 $c\left(u_{2}\right)=3$. 考虑下面两种子情形:

$(2.2 \mathrm{a}) c\left(x_{2}\right) \neq c\left(x_{4}\right)$.

如果 $c\left(u_{1}\right)=1$ 或 $c\left(u_{7}\right)=1$, 改染 $u_{4}$ 为 $3, u_{5}$ 为 2 , 然后给 $u_{3}$ 染 2 . 否则, 改染 $u_{4}$ 为 3 , 然后给 $u_{3}$ 染 2 .

$(2.2 \mathrm{~b}) c\left(x_{2}\right)=c\left(x_{4}\right)$.

由 $c$ 的定义, $c\left(x_{2}\right)=c\left(x_{4}\right)=3$. 首先, 假设 $c\left(x_{5}\right)=1$. 如果 $c\left(u_{1}\right)=1$ 或 $c\left(u_{7}\right)=1$, 改染 $x_{4}$ 为 $2, u_{4}$ 为 $3, u_{5}$ 染 2 , 然后给 $u_{3}$ 染 2 . 如果 $c\left(u_{1}\right)=c\left(u_{7}\right)=2$, 改染 $x_{4}$ 为 $2, u_{4}$ 为 3 , 然后 给 $u_{3}$ 染 2 . 接下来, 假设 $c\left(x_{5}\right)=2$. 如果 $c\left(u_{1}\right)=2$ 或 $c\left(u_{7}\right)=2$, 改染 $x_{4}$ 为 $1, x_{3}$ 为 $2, u_{4}$ 染 3 , 然后给 $u_{3}$ 染 1 . 如果 $c\left(u_{1}\right)=c\left(u_{7}\right)=1$, 改染 $x_{4}$ 为 $1, x_{3}$ 为 $2, u_{4}$ 为 3 , 然后给 $u_{3}$ 染 2 .

这样就得到了 $G$ 的一个线性 3-染色, 这与 $G$ 的选取矛盾! 断言证毕.

断言 5.3 不存在一个 3 -点相邻一个类型 I 2-点同时相邻两个类型 II 2-点.

证明 假设 $G$ 包含 $v_{4}$ 相邻一个类型 I 2-点 $v_{3}$ 和两个类型 II 2-点 $v_{5}$ 和 $v_{7}$. 设 $v_{2}, v_{6}, v_{8}$ 分别是 $v_{3}, v_{5}, v_{7}$ 不同于 $v_{4}$ 的邻点. 设 $v_{1}$ 是 $v_{2}$ 的不同于 $v_{3}$ 的邻点. 设 $z_{1}$ 和 $z_{2}$ 是 $v_{6}$ 的不 同于 $v_{5}$ 的邻点. 设 $y_{1}$ 和 $y_{2}$ 是 $v_{8}$ 的不同于 $v_{7}$ 的邻点. 如图 4 所示.

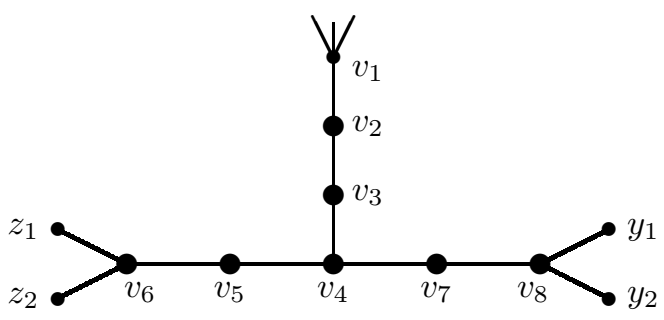

图 4 断言 5.3 中的子结构

由 $G$ 的极小性, $G-v_{3}$ 有一个线性 3-染色 $c$ 应用颜色集合 $C=\{1,2,3\}$. 如果 $v_{2}$ 和 $v_{4}$ 染相同的颜色, 设 $c\left(v_{2}\right)=c\left(v_{4}\right)=1$. 假设 $c\left(v_{1}\right)=2$. 如果 $v_{5}$ 和 $v_{3}$ 中至多有一个染 3 , 则仍 然给 $v_{3}$ 染 3 . 如果 $c\left(v_{5}\right)=c\left(v_{7}\right)=3$, 给 $v_{3}$ 染 2 .

假设 $v_{2}$ 和 $v_{4}$ 染不同的颜色, 设 $c\left(v_{4}\right)=1, c\left(v_{2}\right)=2$. 如果 $v_{5}$ 和 $v_{3}$ 中至多有一个染 3 , 则给 $v_{3}$ 染 3 . 假设 $c\left(v_{5}\right)=c\left(v_{7}\right)=3$. 如果 $v_{6}$ 和 $v_{8}$ 中至多有一个染 2 , 我们给 $v_{3}$ 染 1 , 然后 改染 $v_{4}$ 为 2 . 假设 $c\left(v_{6}\right)=c\left(v_{8}\right)=2$. 于是, $y_{1}$ 和 $y_{2}$ 中至少一个染 1 , 并且 $z_{1}$ 和 $z_{2}$ 中至少 一个染 1 . 根据 $v_{1}$ 的颜色, 考虑下面两种情形:

假设 $c\left(v_{1}\right)=3$. 如果 3 在 $y_{1}, y_{2}, z_{1}, z_{2}$ 上最多出现一次, 我们给 $v_{3}$ 染 1 , 改染 $v_{4}$ 为 2 . 否则, $\left\{c\left(y_{1}\right), c\left(y_{2}\right)\right\}=\left\{c\left(z_{1}\right), c\left(z_{2}\right)\right\}=\{1,3\}$, 染 (或改染) $v_{4}$ 为 $2, v_{3}$ 和 $v_{7}$ 为 1 . 
假设 $c\left(v_{1}\right)=1$. 如果 $3 \in c\left(z_{1}\right), c\left(z_{2}\right)$, 则给 $v_{3}$ 染 3 , 然后改染 $v_{5}$ 为 $1, v_{4}$ 为 2 . 如果 $3 \in c\left(z_{1}\right), c\left(z_{2}\right)$, 则讨论同上. 如果 $c\left(y_{1}\right)=c\left(y_{2}\right)=c\left(z_{1}\right)=c\left(z_{2}\right)=1$, 则染 $v_{3}$ 为 1 , 然后改染 $v_{4}$ 为 2 .

这样就得到 $G$ 的一个线性 3-染色, 这与 $G$ 的选取矛盾, 断言证毕.

断言 5.4 不存在一个 3 -点相邻一个 2 -点同时相邻两个类型 I 2-点.

证明 假设 $G$ 包含 $u_{4}$ 相邻一个 2 -点 $u_{8}$ 和两个类型 I 2 -点 $u_{3}$ 和 $u_{5}$. 设 $u_{2}, u_{6}, u_{9}$ 分别 是 $u_{3}, u_{5}, u_{8}$ 不同于 $u_{4}$ 的邻点. 设 $u_{1}$ 是 $u_{2}$ 的不同于 $u_{3}$ 的邻点. 设 $u_{7}$ 是 $u_{6}$ 的不同于 $u_{5}$ 的邻点. 如图 5 所示.

由 $G$ 的极小性, $G-u_{3}$ 有一个线性 3-染色 $c$ 应用颜色集合 $C=\{1,2,3\}$. 考虑下面两种 情形:

情形 $1 \quad c\left(u_{8}\right) \neq c\left(u_{5}\right)$.

如果 $c\left(u_{2}\right)=c\left(u_{4}\right)$, 则用 $C \backslash\left\{c\left(u_{1}\right), c\left(u_{2}\right)\right\}$ 中颜色染 $u_{3}$. 如果 $c\left(u_{2}\right) \neq c\left(u_{4}\right)$, 则用 $C \backslash\left\{c\left(u_{2}\right), c\left(u_{4}\right)\right\}$ 中颜色染 $u_{3}$.

情形 $\mathbf{2} \quad c\left(u_{8}\right)=c\left(u_{5}\right)=1$.

不失一般性, 假设 $c\left(u_{4}\right)=2$.

子情形 $2.1 \quad c\left(u_{6}\right)=2$.

如果 $c\left(u_{2}\right) \in\{1,2\}$, 则给 $u_{3}$ 染 3 . 如果 $c\left(u_{2}\right)=3$, 则改染 $u_{5}$ 为 3 , 然后给 $u_{3}$ 染 1 .

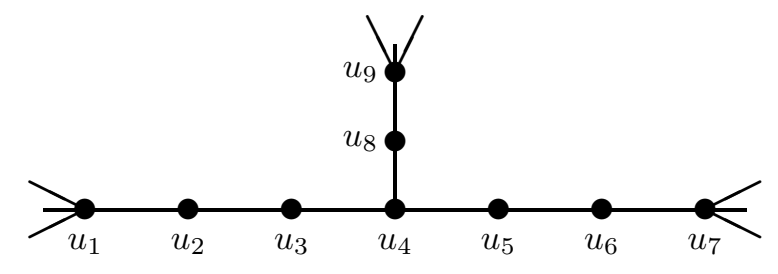

图 5 断言 5.4 中的子结构

子情形 $\mathbf{2 . 2} \quad c\left(u_{6}\right)=3$.

如果 $c\left(u_{2}\right) \in\{1,2\}$, 则给 $u_{3}$ 染 3 . 假设 $c\left(u_{2}\right)=3$. 如果 $c\left(u_{1}\right)=1$ 或 $c\left(u_{7}\right)=1$, 则改染 $u_{5}$ 为 $2, u_{4}$ 为 3 , 然后给 $u_{3}$ 染 2 . 如果 $c\left(u_{1}\right)=c\left(u_{7}\right)=2$, 则改染 $u_{5}$ 为 $2, u_{4}$ 为 3 , 然后给 $u_{3}$ 染 1 . 断言证毕.

令等式 $(*)$ 中 $k=13$. 定义一个初始权函数 $w$ : 如果 $v \in V(G)$, 则 $w(v)=11 d(v)-26$; 如果 $f \in F(G)$, 则 $w(f)=2 d(f)-26$. 用 $n_{2}^{*}(v)$ 表示与 $v$ 相邻的类型 I 2-点的个数.

权转移规则如下:

(R5.1) 每一个 4 -点转 $\frac{13}{3}$ 给每一个相邻 2-点.

(R5.2) 设 $v$ 是一个 3 -点.

如果 $n_{2}^{*}(v)=2$, 则 $v$ 转 $\frac{65}{16}$ 给相邻的类型 I 2-点;

如果 $n_{2}^{*}(v) \leqslant 1$, 则 $v$ 转 $\frac{17}{4}$ 给可能相邻的类型 I 2-点, $\frac{9}{4}$ 给相邻的类型 II 2-点, $\frac{5}{4}$ 给相邻 的满足 $n_{2}^{*}(u)=2$ 的 3 -点 $u$.

设 $w^{\prime}$ 为权转移结束后的新权函数. 
设 $f \in F(G)$, 则 $d(f) \geqslant 13$ 且 $w^{\prime}(f)=w(f)=2 d(f)-26 \geqslant 0$.

设 $v \in V(G)$, 则 $2 \leqslant d(v) \leqslant 4$. 如果 $d(v)=4$, 则 $w(v)=18$ 且 $v$ 至多和 4 个 2-点或 3-点 相邻. 因此, 由 (R5.1),

$$
w^{\prime}(v) \geqslant 18-4 \times \frac{13}{3}=\frac{2}{3} .
$$

假设 $d(v)=3$, 则 $w(v)=11 d(v)-26=33-26=7$. 设 $x, y, z$ 为 $v$ 的邻点且满足 $d(x) \leqslant d(y) \leqslant d(z)$. 由断言 $5.4, x, y, z$ 中最多有两个是类型 I 2-点. 考虑下面 3 种情形:

(a) $n_{2}^{*}(v)=2$. 不失一般性, 假设 $x$ 和 $y$ 是和 $v$ 相邻的类型 I 2-点. 由断言 $5.4, d(z) \geqslant 3$. 如果 $d(z)=4$, 则由 (R5.1), $z$ 转 $\frac{13}{3}$ 给 $v$. 所以由 (R5.2),

$$
w^{\prime}(v) \geqslant 7+\frac{13}{3}-2 \times \frac{65}{16}=\frac{77}{24} .
$$

如果 $d(z)=3$, 则由断言 5.2 和 $(\mathrm{R} 5.2), n_{2}^{*}(z) \leqslant 1$ 且 $z$ 转 $\frac{5}{4}$ 给 $v$. 所以

$$
w^{\prime}(v) \geqslant 7+\frac{5}{4}-2 \times \frac{65}{16}=\frac{1}{8} .
$$

(b) $n_{2}^{*}(v)=1$. 假设 $x$ 是一个和 $v$ 相邻的类型 I 2-点. 如果 $y$ 和 $z$ 都不是类型 II 2-点, 则由 (R5.2),

$$
w^{\prime}(v) \geqslant 7-\frac{17}{4}-2 \times \frac{5}{4}=\frac{1}{4} .
$$

否则, 假设 $y$ 是类型 II 2-点. 由断言 5.2 和 $5.3, z$ 既不是类型 II 2-点, 也不是满足 $n_{2}^{*}(z)=2$ 的 3-点. 由 (R5.2), $v$ 转 $\frac{17}{4}$ 给 $x, \frac{9}{4}$ 给 $y$, 所以

$$
w^{\prime}(v)=7-\frac{17}{4}-\frac{9}{4}=\frac{1}{2} .
$$

(c) $n_{2}^{*}(v)=0$. 由 (R5.2), $v$ 至多转 $\frac{9}{4}$ 给每一个相邻的点, 所以

$$
w^{\prime}(v) \geqslant 7-3 \times \frac{9}{4}=\frac{1}{4} .
$$

假设 $d(v)=2$, 则 $w(v)=-4$. 由断言 $5.1, v$ 至少与一个度数大于 2 的点相邻. 如果 $v$ 和一个 4 -点相邻, 则由 (R5.1),

$$
w^{\prime}(v) \geqslant-4+\frac{13}{3}=\frac{1}{3} .
$$

如果 $v$ 和两个 3 -点相邻, 即 $v$ 是类型 II, 则

$$
w^{\prime}(v) \geqslant-4+\frac{9}{4}+\frac{9}{4}=\frac{1}{2} .
$$

如果 $v$ 和一个 3 -点及一个 2 -点相邻, 即 $v$ 是类型 I, 则由 (R5.2), 3-点转 $\frac{17}{4}$ 给 $v$, 所以,

$$
w^{\prime}(v) \geqslant-4+\frac{17}{4}=\frac{1}{4} \text {. }
$$

这样我们证明了对于所有的 $v \in V(G)$, 有 $w^{\prime}(v)>0$; 所有的 $f \in F(G)$, 有 $w^{\prime}(f) \geqslant 0$, 故 $w^{\prime}$ 满足 (I) 和 (II). 证毕.

\section{参考文献}

1 Yuster R. Linear coloring of graphs. Discret Math, 185: 293-297 (1998)

2 Grünbaum B. Acyclic colorings of planar graphs. Israel J Math, 41: 390-408 (1973)

3 Esperet L, Montassier M, Raspaud A. Linear choosability of graphs. Discret Math, 308: 3938-3950 (2008)

4 Raspaud A, Wang W. Linear coloring of planar graphs with large girth. Discret Math, doi: 10.1016/j.disc. 2008.04.032 